\title{
Dementia Community Screening Program in District Health Area 11: Phase 1
}

\section{Tharin PHENWAN ${ }^{1,2, *}$, Weeratian TAWANWONGSRI ${ }^{1}$, Phanit KOOMHIN $^{1}$ and Udomsak SAENGOW ${ }^{1}$}

\author{
${ }^{1}$ School of Medicine, Walailak University, Nakhon Si Thammarat 80160, Thailand \\ ${ }^{2}$ School of Health Sciences, University of Dundee, Dundee, UK
}

("Corresponding author's e-mail: tharin.ph@wu.ac.th, t.phenwan@dundee.ac.uk)

Received: 5 June 2018, Revised: 14 November 2018, Accepted: 20 December 2018

\begin{abstract}
To estimate the prevalence of dementia among Thai elderly in the upper Southern region of Thailand, we performed a cross-sectional screening of all Thai older people from 2 areas of Nakhon Si Thammarat province: Tambon Baan Thungchon, Tha Sala district, and Moo 6 and 7 from Sichon district, from December 2016 to November 2017. Trained health volunteers identified the participants in their communities and collected data including age, gender, comorbidities, Timed Up and Go Test (TUGT) results, and Montreal Cognitive Assessment (MoCA) scores. Our sample comprised 773 participants, of which 605 (78.3\%) were from Baan Thungchon area, while 168 were from Moo 6 and Moo 7 of Sichon district. The majority of participants were female $(431,55.7 \%)$. The mean age of the participants was 79 \pm 9.1 years, with a minimum age of 60 , and a maximum age of 95 . Their comorbidities were hypertension $(42.9 \%)$, type II diabetic mellitus (33.2\%), dyslipidemia $(37.5 \%)$, and osteoarthritis of the knees $(35.8$ $\%$ ). $35.1 \%$ of them also had positive TUGT. Sixty-seven participants $(8.7 \%)$ scored 7 or lower in the Abbreviated Mental Test (AMT). Five participants (7.5\%) had a positive screening for dementia.
\end{abstract}

Keywords: Elderly, Dementia, Screening, Prevalence

\section{Introduction}

As a result of declining fertility rates and longer life expectancy, the Thai population is aging rapidly. In 2016, $16.6 \%$ of Thais, an estimated 11.2 million people, were 60 years or older, compared to approximately $5 \%$ in 1995 [1]. With more than a quarter of the population being elderly, Thailand will have the $2^{\text {nd }}$ highest percentage of aging population in Southeast Asia, with numbers rising to approximately 22.6 million people, within the next 15 years.

An increasing non-communicable disease (NCD) that also increases with the ageing population is dementia. Dementia is an umbrella term for a chronic condition that impairs the elderly's cognitive functions, and is particularly prevalent in low-to-middle income countries [2,3]. It is one of the most common chronic medical conditions, and has become a main concern in health care due to its impact on functional impairment, high-mortality rate, and effects on carers [4,5].

The $10^{\text {th }}$ revision of the International Statistical Classification of Diseases and Related Health Problems (ICD-10) defined dementia as "a syndrome due to disease of the brain, usually of a chronic or progressive nature, in which there is disturbance of multiple higher cortical functions, including memory, thinking, orientation, comprehension, calculation, learning capacity, language, and judgment". While a clear etiology of dementia is not well-established, there are several risk factors for dementia, including advanced age, family history of dementia, history of trauma or toxin exposures, smoking, sedentary lifestyle, poor nutritional status, diabetes mellitus (DM), history of previous myocardial infarction, and 
http://wjst.wu.ac.th

history of stroke [6,7]. Many conditions can cause dementia, such as Alzheimer's, Lewy body, vascular and Parkinson's disease, and HIV infection. For the convergence of clinical practice and research, the diagnostic criteria were recently revised and launched in the $5^{\text {th }}$ edition of the American Psychiatric Association's Diagnostic and Statistical Manual (DSM-5) [8]. In Thailand, the guidelines emphasize the importance of dementia screening, with general practitioners (GPs) assessing the elderly with dementia screening tools.

The World Health Organization (WHO) reported that the standardized prevalence of dementia in Southeast Asia was $6.4 \%$ for those aged over 60 [7]. As for dementia prevalence in Thailand, 1 multistage random cross-sectional survey study conducted by the Ministry of Public Health of Thailand in 1997 revealed that $3.3 \%$ of the elderly had dementia [9]. Other studies revealed that the prevalence of dementia was $2.35-2.86 \%, 12.7$ and $7.7 \%$ in the Northern region, Bangkok, and the North-eastern region, respectively [10-13]. However, no up-to-date research has surveyed the exact prevalence of dementia particular to the upper Southern region of Thailand yet. This study aims to investigate the present status of the prevalence of dementia among elderly Thais in this area.

\section{Materials and methods}

\section{Design and study settings}

A cross-sectional survey design was used. From December 2016 to November 2017, we screened all elderly people from 2 areas of Nakhon Si Thammarat province: Tambon Baan Thungchon, Tha Sala district, and Moo 6 and 7 from Sichon district. Health volunteers in those areas received a half-day training course with the principal investigator (TP) to screen for dementia using an Abbreviated Mental Test (AMT) to ensure that these health volunteers could use this screening tool correctly.

After that, the health volunteers went to their respective areas to screen the participants in their communities. Those who scored less than 7 on AMT would be referred to see the investigators (TP or WT) for further screening using the Thai version of the Montreal Cognitive Assessment (MoCA). After the 2nd screening with the MoCA, those who had positive results would be referred for further investigation for the etiology of their abnormal screening test by PK.

\section{Sample}

We used the WHO's report to calculate the sample size for proportionality with a $2 \%$ margin of error to represent the prevalence of the dementia population in Nakhon Si Thammarat province and used a cluster sampling method to select designated areas for the screening [7]. From the calculation, the number of participants that were needed was 566, and allowing $10 \%$ for missing participants gave a total number of 623. Inclusion criteria were: any individual who was older than 60 years old, lived in Nakhon Si Thammarat province, could communicate in Thai, and had not been diagnosed with any psychiatric disorders. Exclusion criterion was unwillingness to participate.

\section{Instrument}

The health volunteers collected demographic data. Data on underlying diseases were gathered from medical records. In the case of missing data or new cases, health volunteers would ask about participants' underlying diseases directly.

\section{Abbreviated Mental Test (AMT)}

The AMT is a screening tool developed to assess the elderly for the possibility of dementia. It is recommended by the Institute of Geriatric Medicine due to its practical use. The screening tool consists of 10 questions, and a score of lower than 8 suggests the possibility of dementia [14]. The Thai version of the AMT is also used widely among healthcare workers.

\section{The Montreal Cognitive Assessment (MoCA)}

The MoCA is a brief screening test designed to detect cognitive impairment in older adults and is also available in Thai language. It is more sensitive for the detection of mild cognitive impairment. The 
http://wjst.wu.ac.th

included items cover a wider range in terms of cognitive domains, including memory, language, attention, visuospatial, and executive functions [15].

\section{Turn Up and Go Test (TUGT)}

The TUGT is a "well-established, uncomplicated, and quickly administrated test of one movement sequence: starting from a sitting position in an armchair, standing up and walking three meters, turning around, walking back to the chair, and sitting down again" [16].

\section{Data analysis}

The statistical analysis was performed using SPSS software version 17 (SPSS Inc., Chicago, IL, USA). Mean and standard deviation (SD) or median and range were used to describe continuous data. Frequency and percentage were used for categorical data.

\section{Ethical consideration}

The Human Research Ethics Committee of Walailak University approved this study (WUEC 16/085). The study complied with the International Conference on Harmonization of Good Clinical Practice and principles of the Declaration of Helsinki.

\section{Results and discussion}

The response rate was $79.7 \%$. There were 970 eligible elderly people from the record within our designated areas, and 773 participants joined our study. The main reasons for non-participation were frailty and a failure to understand the questions. 605 (78.2 \%) came from Tha Sala district, while 168 came from Moo 6 and Moo 7 of Sichon district. The majority of participants were female (431, 55.7 \%). The mean age of the participants was $79 \pm 9.1$ years, with a minimum age of 60 and a maximum age of 95. Their comorbidities were hypertension (42.9\%), type II DM (33.2 \%), dyslipidemia $(37.5 \%)$, and osteoarthritis of the knees $(35.8 \%) .35 .1 \%$ of them also had a positive TUGT. Sixty-seven participants $(8.7 \%)$ scored 7 or lower on the AMT (Table 1).

Table 1 Participants' characteristics.

\begin{tabular}{lc}
\hline Characteristics & $\mathbf{n}=\mathbf{7 7 3}$ \\
\hline Gender & $431(55.7)$ \\
Female & $342(44.3)$ \\
Male & $79 \pm 9.1$ \\
\hline Age (years) & \\
\hline Education level & $562(72.7)$ \\
Primary & $196(25.4)$ \\
Secondary & $15(1.9)$ \\
Tertiary & $332(42.9)$ \\
\hline Underlying diseases & $257(33.2)$ \\
Hypertension & $290(37.5)$ \\
Type II Diabetes Mellitus & $277(35.8)$ \\
Dyslipidemia & \\
Osteoarthritis of the knees & $271(35.1)$ \\
\hline Timed Up and Go Test (TUGT) & $502(64.9)$ \\
Positive & $67(8.7)$ \\
Negative & $706(91.3)$ \\
\hline AMT scores & \\
Less or equal than 7 & \\
More than 7 &
\end{tabular}


http://wjst.wu.ac.th

Participants who scored 7 or lower on the AMT received a 2nd screening with the MoCA. In Table 2, the majority was female $(56.7 \%)$ with a mean age of $70 \pm 6.7$. Their comorbidities were hypertension (58.2\%), type II DM (53.7\%), dyslipidemia (47.7\%), and osteoarthritis of the knees (32.8\%). Five participants $(7.5 \%)$ had a positive screening for dementia.

Table 2 Participants with abnormal screening test characteristics.

\begin{tabular}{lc}
\hline Characteristics & $\mathbf{n}=\mathbf{6 7}$ \\
\hline Gender & $38(56.7)$ \\
$\quad$ Female & $29(43.3)$ \\
$\quad$ Male & $70 \pm 6.7$ \\
Age (years) & \\
Education level & $52(77.6)$ \\
$\quad$ Primary & $15(22.4)$ \\
$\quad$ Secondary & \\
Underlying diseases & $39(58.2)$ \\
$\quad$ Hypertension & $36(53.7)$ \\
$\quad$ Type II Diabetes Mellitus & $32(47.7)$ \\
$\quad$ Dyslipidemia & $22(32.8)$ \\
$\quad$ Osteoarthritis of the knees & \\
Timed Up and Go Test (TUGT) & $12(17.9)$ \\
$\quad$ Positive & $55(82.1)$ \\
$\quad$ Negative & \\
MoCA-T & $5(7.5)$ \\
$\quad$ Positive & $62(92.5)$ \\
$\quad$ Negative &
\end{tabular}

\section{Discussion}

This study is the 1st phase of our work to assess dementia prevalence in upper Southern Thailand. We selected 2 areas as the pilot areas. Screening results showed that the prevalence of dementia in our participants, $7.5 \%$, was higher than the WHO's estimation of $6.4 \%$ [7], and also higher than the national prevalence of $5 \%$ [1]. We will continue to investigate the cause of the high prevalence of this area in our future study. Given that Thailand will become a super-aged society in the next 20 years, early dementia detection can be beneficial for healthcare providers and patients, given the multiple modalities needed to care for this group of patients: pharmacological treatments and non-pharmacological treatments, patient education about self-care, nutrition, risk factors control, disease progression, exercise programs, and carer training [17]. Preparation could also be made for rehabilitation to ensure that the patients would receive optimal quality of life and, ideally, Advance Care Planning (ACP) about their preferred care when patients became terminal [18]. In addition, several etiologies of dementia, for example, hematoma, meningitis, depression, etc., are reversible [19]. Thus, with early detection and treatment of curable causes, we could reduce the disease burden on the hospital and the family drastically.

The strength of this study is that it is the $1^{\text {st }}$ study to perform community screening in Nakhon Si Thammarat. No data had been collected in this area before. Furthermore, we have a large number of participants as a consequence of going directly into the community rather than having them come to the hospital. This strategy was beneficial, as shown in the high response rate $(79.7 \%)$. We also used double screening methods with health volunteers rather than doctors to ensure that the screening was more reliable. Finally, we detected more underlying diseases in this group of elderly people as well, since many of them did not receive their care from the public health service, i.e., they went to private practices or had 
http://wjst.wu.ac.th

alternative treatment with herbalists or traditional doctors. With this screening program, we could find more data on these missing groups.

Our work has several limitations. Firstly, we only selected 2 sub-district areas, which cannot reflect actual prevalence in the whole of Nakhon Si Thammarat province. Secondly, comorbidities of participants were retrieved from medical records or asked about directly by the health volunteers. It is very highly probable that there were some undetected diseases or other missing data.

To further our findings in future work, we aim to perform dementia screening in other sub-districts and follow up with those who already tested positive with the MoCA to determine the etiologies of their abnormal screening tests.

\section{Conclusions}

The AMT and MoCA screening tools showed that the prevalence of elderly people in Nakhon $\mathrm{Si}$ Thammarat, Thailand who are susceptible to dementia is $7.5 \%$, which is higher than the national rate. We will continue to investigate for further causes of this finding.

\section{Recommendations and further study}

1. Strategies should be suggested to prepare families to take care of dementia patients so that they will receive optimal care with a good quality of life.

2. Future research will focus on getting more examples from other areas and finding treatable etiologies of dementia to reduce the burden of care for families and healthcare personnel.

\section{Acknowledgements}

We would like to thank Associate Professor David Harding and Mr. Alan Duncan for their help with English language editing.

\section{References}

[1] National Statistical Office, Statistical Yearbook Thailand. In: SF Bureau (Ed.). Bangkok Block, Bangkok, 2017, p. 21.

[2] N Lancet. Societies can both grow old and lower dementia burden. Lancet Neurol. 2015; 14, 967.

[3] GM McKhann, DS Knopman, H Chertkow, BT Hyman, CRJ Jack, CH Kawas, WE Klunk, WJ Koroshetz, JJ Manly, R Mayeux, RC Mohs, JC Morris, MN Rossor, P Scheltens, MC Carrillo, B Thies, S Weintraub and $\mathrm{CH}$ Phelps. The diagnosis of dementia due to Alzheimer's disease: Recommendations from the National Institute on Aging-Alzheimer's association workgroups on diagnostic guidelines for Alzheimer's disease. Alzheimers. Dement. 2011; 7, 263-9.

[4] P Mekawichai and S Saetang. Carer burden among Thai dementia patients' carers. J. Med. Assoc. Thai. 2013; 58, 101-10.

[5] P Siritipakorn and W Muangpaisan. Associated factors of carer burden among family carers of dementia patients in a geriatric clinic, Siriraj Hospital. J. Nurs. Sci. 2015; 38, 54-64.

[6] J Surawan, S Areemit, S Tiamkao, T Sirithanawuthichai and S Saensak. Risk factors associated with post-stroke dementia: A systematic review and meta-analysis. Neurol. Int. 2017; 9, 7216.

[7] World Health Organization. Dementia: A public health priority, Available at: https://www.who.int/mental_health/publications/dementia_report_2012/en, accessed January 2018.

[8] PS Sachdev, A Mohan, L Taylor and DV Jeste. DSM-5 and mental disorders in older Individuals: An overview. Harv Rev. Psychiatr. 2015; 23, 320-8.

[9] S Jitapunkul, C Kunanusont, W Phoolcharoen and P Suriyawongpaisal. Prevalence estimation of dementia among Thai elderly: A national survey. J. Med. Assoc. Thai. 2001; 84, 461-7.

[10] S Wangtongkum, P Sucharitkul, N Silprasert and R Inthrachak. Prevalence of dementia among population age over 45 years in Chiang Mai, Thailand. J. Med. Assoc. Thai. 2008; 91, 1685. 
http://wjst.wu.ac.th

[11] T Charernboon, M Phanasathit, S Tangwongchai, S Hemrungrojn and P Worakul. Depression and dementia among members of 15 elderly clubs in Bangkok. Thammasat Med. J. 2010; 10, 428-36.

[12] S Lertkratoke, S Nanthamongkolchai and S Pitikultang. Prevalence and factors associated with dementia among elderly people in Oraphim subdistrict, Khonburi district, Nakhonratchasima province. J. Public Health 2013; 43, 42-54.

[13] S Kumfu, K Chaimongkol, C Chaichompoo and P Kaewmuneewong. Screening of dementia and self-care burden among the elderly in Mae Chan district, Chiang Rai province. Srinagarind Med. J. 2014; 29, 389-93.

[14] ST Pendlebury, SP Klaus, M Mather, MD Brito and RM Wharton. Routine cognitive screening in older patients admitted to acute medicine: Abbreviated mental test score (AMTS) and subjective memory complaint versus montreal cognitive assessment and IQCODE. Age Ageing 2015; 44, 1000-5.

[15] DH Davis, ST Creavin, JL Yip, AH Noel-Storr, C Brayne and S Cullum. Montreal cognitive assessment for the diagnosis of Alzheimer's disease and other dementias. Cochrane Database Syst. Rev. 2015; 2015, CD010775.

[16] Y Cedervall, AM Stenberg, HB Åhman, V Giedraitis, F Tinmark, L Berglund, K Halvorsen, M Ingelsson, E Rosendahl and AC Åberg. Timed Up-and-Go Dual-Task testing in the assessment of cognitive function: A mixed methods observational study for development of the UDDGait protocol. Int. J. Environ. Res. Public Health 2020; 17, 1715.

[17] D Forbes, SC Forbes, CM Blake, EJ Thiessen and S Forbes. Exercise programs for people with dementia. Cochrane Database Syst. Rev. 2015; 2015, CD006489.

[18] KD Jethwa and O Onalaja. Advance care planning and palliative medicine in advanced dementia: A literature review. BJPsych. Bull. 2015; 39, 74-8.

[19] V Solfrizzi, E Scafato, D Seripa, M Lozupone, BP Imbimbo, A D'Amato, R Tortelli, A Schilardi, L Galluzzo, C Gandin, M Baldereschi, AD Carlo, D Inzitari, A Daniele, C Sabba, G Logroscino, F Panza and $\mathrm{G}$ Italian. Longitudinal study on aging working reversible cognitive frailty dementia and all-cause mortality: The Italian longitudinal study on aging. J. Am. Med. Dir. Assoc. 2017; 18, 89e1$89 \mathrm{e} 8$. 